\title{
First-Principles Study of Arsenic Atom, Its Irons and Molecule
}

\author{
N. R. Sapkota ${ }^{1 *}$ and J. J. Nakarmi ${ }^{2}$ \\ ${ }^{1}$ Department of Physics, Prithvi Narayan Campus, Pokhara, Nepal \\ ${ }^{2}$ Central Department of Physics, Tribhuvan University, Kirtipur, Kathmandu, Nepal \\ *Corresponding address: nawraj.sapkota2014@gmail.com
}

\begin{abstract}
In the present work, we have performed the ground state energy calculations for arsenic atom, its ions and molecule using Hartree-Fock (HF) cluster approximation. The correlation effects in the HF calculations have been taken into account by considering the Møller-Plesset second order perturbation (MP2) and truncated form of Configurational Interaction (CI) that includes single, double, and quadruple excitations, also known as QCISD implemented by the Gaussian 03 sets of program. Our study shows that the ground state of arsenic atom is a quadrate state i.e., charge zero and multiplicity four with ground state energy - $60796.66 \mathrm{eV}$ in MP2 levels of calculation with cc-pVDZ basis set. We have performed the first-principles calculation to study the first electron affinity and ionization energies of arsenic atom up to tenth level. The first-principles calculation has been also carried out to study the equilibrium configuration of arsenic molecule (As2). The bond length and binding energy of arsenic molecule (As2) is found to be $2.15 \mathrm{~A}$ and $3.65 \mathrm{eV}$ in MP2 levels of approximation with basis set 6-311G(3df). Our study has been extended to calculate electrostatic potential for arsenic molecule (As2), whose values at global maxima and minima are found to be $0.30 \mathrm{eV}$ and $-0.20 \mathrm{eV}$ respectively. The calculation of HOMO-LUMO energy gap for the arsenic molecule (As2) is almost independent of choice of basis sets as well as levels of approximation. The HF, MP2, and QCISD calculations also have been carried out to estimate the electric field gradient (EFG) parameters for the excited nuclear state in arsenic molecule (As2). Our results show that the HF, MP2, and QCISD values for the EFG parameters do not differ significantly, indicating that electron correlation effects do not contribute for the determination of EFG parameter.
\end{abstract}

Keywords: HF, MP2, DFT, QCISD, HOMO-LUMO energy gap, Electron affinity, Ionization energy

\section{INTRODUCTION}

The electronic configuration of arsenic is $[\mathrm{Ar}] 3 \mathrm{~d}^{10} 4 \mathrm{~s}^{2} 4 \mathrm{p}^{3}$, so it is a p-block element. Arsenic occurs free in nature, but is most often found in the minerals arsenopyrite (FeAsS), realgar (AsS) and orpiment $\left(\mathrm{As}_{2} \mathrm{~S}_{3}\right)$. Arsenic is mainly obtained by the heating of arsenic containing sulfides. The most commercial arsenic is obtained by heating arsenopyrite. Arsenic appears in three allotropic forms: yellow, black and gray; with gray being the most common. Arsenic is a highly poisonous metalloid. Since it is a metalloid, arsenic has a high density, moderate thermal conductivity, and limited ability to conduct electricity. The oxide of arsenic is amphoteric which means it can act as both an acid and a base. The oxidation states of arsenic are $+5,+3,+2,+1$ and -3 . The most important use of arsenic is in the preservation of wood. Many of its compounds are deadly poison and used as insecticides, weed killer and rat poison. Minute amounts of arsenic are used in the electronics industry. It is added to germanium and silicon to make transistors. A compound of arsenic, gallium arsenide ( $\mathrm{GaAs}$ ), is also used to make light-emitting diodes (LEDs). LEDs produce the lighted numbers in hand-held calculators, clocks, watches, and a number of other electronic devices. Some compounds, called arsenides, are used in the manufacture of paints, wallpapers, and ceramics. Arsenic and its compounds are toxic to animals. In low doses, arsenic produces nausea, vomiting, and diarrhea. In larger doses, it causes abnormal heart beat, damage to blood vessels, and a feeling of "pins and needles" in hands and feet. Small corns or warts may begin to develop on the palms of the hands and the soles of the feet. Direct contact with the skin can cause redness and swelling. Long term exposure to arsenic and its compounds can cause cancer. Inhalation can result in lung cancer. If swallowed, cancer is likely to develop in the bladder, kidneys, liver, and lungs. In large doses, arsenic and its compounds can cause death.

In our best knowledge no calculation have been reported whose main point of study is the ground state energy, electron affinity and ionization energies of arsenic atom. In literature, we found only the value of first ionization energy of arsenic atom to be $9.7886 \mathrm{eV}$ [1], whereas its value in our calculation is $9.83 \mathrm{eV}$. Also we found different values of bond length of arsenic molecule $\left(\mathrm{As}_{2}\right)$ as $2.50 \AA$ [2], $2.10 \AA$ [3] and $2.299 \AA$ [4], whereas the experimental value of bond length of arsenic molecule $\left(\mathrm{As}_{2}\right)$ is 2.44 [5]. Due to the different calculated values of bond length with significant deviation from experimental value, we are interested in finding the bond length as well as binding energy of arsenic molecule $\left(\mathrm{As}_{2}\right)$. Furthermore we found only the HOMO-LUMO energy gap of compounds of arsenic atom [6,7], we focus our study on the HOMO-LUMO energy gap of arsenic molecule $\left(\mathrm{As}_{2}\right)$.

The first-principles methods are being widely used to study the electronic structure of atoms [8]. These methods enable one to 
calculate the various physical properties like the ground state energy, dipole moment, ionization potential, polarizability, nuclear quadrupole moment etc. of a wide range of solid state systems. Furthermore, the physical and chemical properties of large biological systems such as DNA molecules are also being studied using these methods [9]. The first-principles approaches can be classified into three main categories: the Hartree-Fock approach, the density functional approach and the quantum Monte-Carlo approach [10].

A nucleus may possess an electric quadrupole moment. The quadrupole moment $Q$ is a measure of the ellipticity of the distribution of charge in the nucleus. The quadrupole moment may have positive or negative value depending upon the distribution of charge inside the nucleus. An egg shaped nucleus has $Q$ positive, a saucer shaped nucleus has $Q$ negative [11]. Nuclei of spin $I \geq 1$ have an electric quadrupole moment. Due to the ground state $\operatorname{spin} I=3 / 2$, we have calculated the nuclear quadrupole interaction parameters of ${ }^{75} \mathrm{As}$.

\section{METHODOLOGY}

All the calculations in the present work are performed by using the Gaussian 03 sets of program with different levels of approximation such as HF, MP2, DFT(B3LYP) and QCISD using different basis sets [12]. The basis sets used are based on the Gaussian functions and the consistency of the results obtained is tested by their convergence with respect to the basis sets of increasing size and complexity. To calculate the maximum and minimum electrostatic potential within the molecular surface of arsenic molecule $\left(\mathrm{As}_{2}\right)$, the post processing of Gaussian output is performed by multiwfn 3.2.1set of program.

Knowing the ground state energy of arsenic atom and its ion $\mathrm{As}^{-}$, we have estimated the electron affinity for the arsenic atom with the aid of following relation:

$$
\text { E.A. }=\mathrm{E}(\mathrm{As})-\mathrm{E}\left(\mathrm{As}^{-}\right)
$$

where E.A. denote the electron affinity, E(As) and E(As-) denote the ground state energy of the Arsenic atom(As) and the ion As' respectively.

Also knowing the ground state energy of the arsenic atom and its ions $\mathrm{As}^{+}$to $\mathrm{As}^{+10}$, we have estimated the values of the ionization energies of the arsenic atom by using the following relation:

$$
\delta \mathrm{E}_{\mathrm{i}}=\mathrm{E}\left(\mathrm{As}^{+\mathrm{i}}\right)-\mathrm{E}\left(\mathrm{As}^{+\mathrm{j}}\right)
$$

where, $\delta \mathrm{E}_{\mathrm{i}}$ represents the $\mathrm{i}^{\text {th }}$ ionization energy, $\mathrm{E}\left(\mathrm{As}^{+\mathrm{i}}\right)$ represents the total energy value of arsenic atom at the $i^{\text {th }}$ level of ionization with index $i=1,2, \quad \ldots, 10$ and the index $j=i-1$. We have estimated the binding energy of the arsenic molecule $\left(\mathrm{As}_{2}\right)$ with the help of the following relation:

$$
\text { B.E. }=2 \mathrm{E}(\mathrm{As})-\mathrm{E}(\mathrm{As}-\mathrm{As})
$$

where, $E(A s)$ and $E(A s-A s)$ denote the ground state energy of arsenic atom (As) and arsenic molecule $\left(\mathrm{As}_{2}\right)$ respectively.

\section{RESULTS AND DISCUSSION}

In the present work, we have studied; the ground state energy of the arsenic atom and its ions, the electron affinity and the ionization energy of the arsenic atom, the binding energy and bond length of the arsenic molecule $\left(\mathrm{As}_{2}\right)$, electrostatic potential of arsenic molecule $\left(\mathrm{As}_{2}\right)$, Basis Set Superimposition Error on binding energy of arsenic molecule $\left(\mathrm{As}_{2}\right)$ using counterpoise method with different basis sets and different level of approximations, HOMO-LUMO energy gap with related parameters in the arsenic molecule $\left(\mathrm{As}_{2}\right)$ and the electric field gradient parameters for the excited nuclear state in arsenic molecule $\left(\mathrm{As}_{2}\right)$. These calculations have been carried out by using HF, MP2, DFT(B3LYP) and QCISD levels of approximation with different basis sets.

\section{(i) Ground state energy, Electron affinity and Ionization energies of arsenic atom}

The HF, MP2, DFT(B3LYP), and QCISD levels of calculation for the ground state energy of arsenic atom and its ion have been performed using different basis sets. Our result shows that the calculated value of the ground state energy of the arsenic atom and its ions get lowered on increasing the size of the basis sets in all level of approximations. However, the energy values obtained follows the following sequence,

$$
\mathrm{E}_{\mathrm{HF}}>\mathrm{E}_{\mathrm{HF}+\mathrm{MP} 2}>\mathrm{E}_{\mathrm{QCISD}}>\mathrm{E}_{\mathrm{DFT}(\mathrm{B} 3 \mathrm{LYP})}
$$

We have calculated the electron affinity of arsenic atom using HF, MP2 and QCISD levels of approximation with different basis sets. The basis set 6-31G in the MP2 levels of approximation predicts the value of electron affinity for arsenic atom to be $-0.81 \mathrm{eV}$, which is within $1.3 \%$ as compared to the experimental value of $-0.80 \mathrm{eV}[5]$.

We have also estimated the values of the ionization energies for the arsenic atom by considering different levels of ionization i.e. $\mathrm{As}^{+}$to $\mathrm{As}^{+10}$. The MP2 values of the first to tenth ionization energies for the arsenic atom have been estimated to be $9.83 \mathrm{eV}, 18.30 \mathrm{eV}, 27.64 \mathrm{eV}, 48.14 \mathrm{eV}, 61.03 \mathrm{eV}, 123.96 \mathrm{eV}$, $157.17 \mathrm{eV}, 191.60 \mathrm{eV}, 234.80 \mathrm{eV}$ and $257.38 \mathrm{eV}$ respectively. Our estimated values of the first to tenth ionization energies for the arsenic atom agree to the corresponding experimental values of $9.82 \mathrm{eV}, 18.64 \mathrm{eV}, 28.35 \mathrm{eV}, 50.13 \mathrm{eV}, 62.62 \mathrm{eV}$, $127.53 \mathrm{eV}, 159.61 \mathrm{eV}, 195.89 \mathrm{eV}, 234.23 \mathrm{eV}$ and $273.62 \mathrm{eV}$ respectively within $6 \%$. This shows that our estimated values of the first to tenth ionization energies for the arsenic atom are as expected and within the acceptable limit as compared to the experimental values.

\section{(ii) Binding energy and Bond length of arsenic molecule $\left(\mathrm{As}_{2}\right)$}

Equilibrium configuration of arsenic molecule $\left(\mathrm{As}_{2}\right)$ has been studied by calculating the binding energy and bond length in all levels of approximation using the different basis sets. The calculated values of the binding energy and the bond length 
for arsenic molecule obtained with the basis set $6-311 \mathrm{G}(3 \mathrm{df})$ is shown in Table 3.1.

Table 3.1: Comparison between the estimated and the experimentally observed values of the binding energy and the bond length for arsenic molecule obtained with the basis set 6-311G(3df).

\begin{tabular}{|c|c|c|c|}
\hline Parameters & $\begin{array}{c}\text { Levels of } \\
\text { approximation }\end{array}$ & $\begin{array}{l}\text { Estimated } \\
\text { Values }\end{array}$ & $\begin{array}{c}\text { Experimental } \\
\text { Values }\end{array}$ \\
\hline \multirow{4}{*}{$\begin{array}{l}\text { Bond length } \\
(\AA)\end{array}$} & HF & 2.06 & \multirow{4}{*}{$2.44[5]$} \\
\hline & MP2 & 2.15 & \\
\hline & DFT(B3LYP) & 2.10 & \\
\hline & QCISD & 2.11 & \\
\hline \multirow{4}{*}{$\begin{array}{l}\text { Binding } \\
\text { energy } \\
(\mathrm{eV})\end{array}$} & $\mathrm{HF}$ & 0.58 & \multirow{4}{*}{$3.61[5]$} \\
\hline & MP2 & 3.65 & \\
\hline & DFT(B3LYP) & 4.06 & \\
\hline & QCISD & 3.19 & \\
\hline
\end{tabular}

We have also performed the Basis Set Superimposition Error calculations using Counterpoise method for various basis sets and level of approximations in ordered to obtain the corrected binding energy. With the basis set $6-311 \mathrm{G}(3 \mathrm{df})$, the MP2 levels of approximation predict the value of binding energy of arsenic molecule as $3.58 \mathrm{eV}$, which is within $0.84 \%$ as compared to the experimental value whereas without counterpoise correction the corresponding binding energy was $3.65 \mathrm{eV}$, which is within $1.11 \%$ of the experimental value 3.61 $\mathrm{eV}$. This shows that the counterpoise method gives more close value of binding energy to the experimental value than the corresponding value without counterpoise method. Thus, we can conclude that while performing atomic calculations it is necessary to account for the BSSE.

We have also studied the variation of the ground state energy of arsenic molecule $\left(\mathrm{As}_{2}\right)$ with respect to the distance (As-As) obtained with the different levels of calculation using the basis set 6-311G(3df). The plot has been presented in Figure 3.2 with the scan coordinate as the distance between two arsenic atom i.e. the As-As separation in the arsenic molecule $\left(\mathrm{As}_{2}\right)$ in MP2 levels of approximation using the basis set 6-311G(3df).

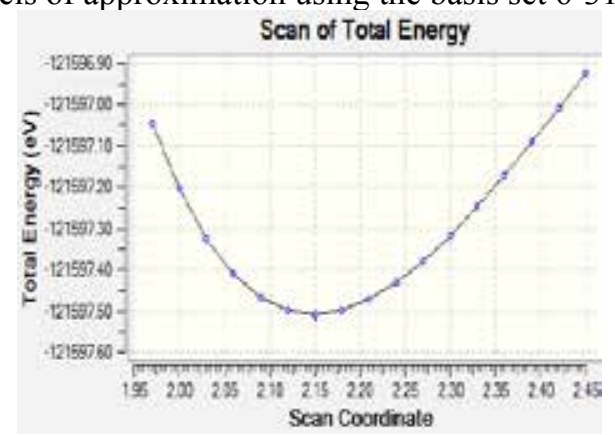

Figure 3.2: Variation of ground state energy $(\mathrm{eV})$ of arsenic molecule $\left(\mathrm{As}_{2}\right)$ with scan co-ordinate as bond length $(\AA)$ in MP2 levels of approximation using the basis set 6-311G(3df).
It is seen from Figure 3.2 that varying the distance (d) between two arsenic atoms from $1.97 \AA$ to $2.45 \AA$, the change in energy of arsenic molecule is about $0.12 \mathrm{eV}$ and the minimum in energy occurs at around $2.15 \AA$.

\section{(iii) Electrostatic of arsenic molecule $\left(\mathrm{As}_{2}\right)$}

We have calculated the electrostatic potential of arsenic molecule $\left(\mathrm{As}_{2}\right)$ in the HF levels of approximation with the choice of basis set 6-311G(3df). The post processing of Gaussian output is performed by multiwfn 3.2.1 set of program to calculate the maximum and minimum electrostatic potential within the molecular surface of arsenic molecule $\left(\mathrm{As}_{2}\right)$. We found 44 maximas and 34 minimas as shown in Figure 3.3. Among them $6^{\text {th }}$ maxima and $23^{\text {th }}$ minima are of global nature. The electrostatic potential of arsenic molecule $\left(\mathrm{As}_{2}\right)$ at global maxima and minima are found to be $0.30 \mathrm{eV}$ and $-0.20 \mathrm{eV}$ respectively.

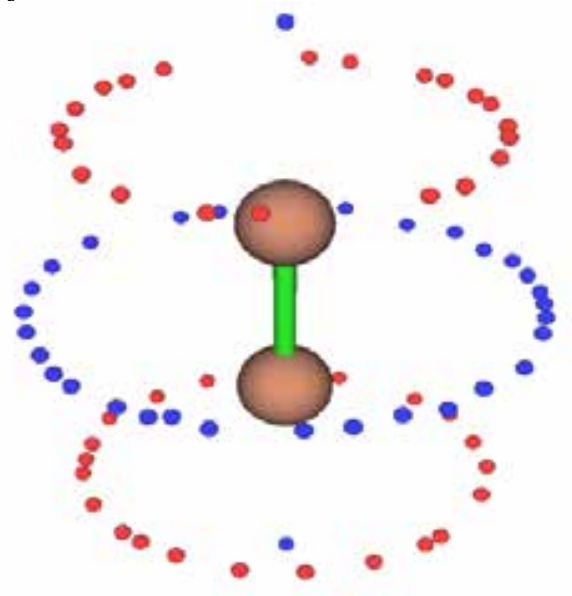

Figure 3.3: maximas and minimas of $\mathrm{As}_{2}$ molecule (red indicate maximas and blue indicate minimas) within the molecular surface of isovalue 0.01 a.u.

(iv) HOMO-LUMO energy gap for molecule ( $\left.\mathrm{As}_{2}\right)$ HOMO stands for highest occupied molecular orbital. LUMO stands for lowest unoccupied molecular orbital. The energy difference between a molecule's HOMO and its LUMO is termed as the HOMO-LUMO energy gap. The energy gap between HOMO and LUMO is a critical parameter in determining molecular electrical transport properties [13-15]. Molecular orbitals (HOMO and LUMO) and their properties such as energy are very useful for physicists and chemists and are very important parameters for quantum chemistry. We have calculated HOMO-LUMO energy gap for the arsenic molecule $\left(\mathrm{As}_{2}\right)$ in HF, MP2 and QCISD levels of approximation using the different basis sets with NBO analysis method.

The HOMO and LUMO in the arsenic molecule ( $\left.\mathrm{As}_{2}\right)$ with MP2 levels of approximation using the basis set $\quad 6-311 \mathrm{G}(3 \mathrm{df})$ is shown in the Figure 3.4. 


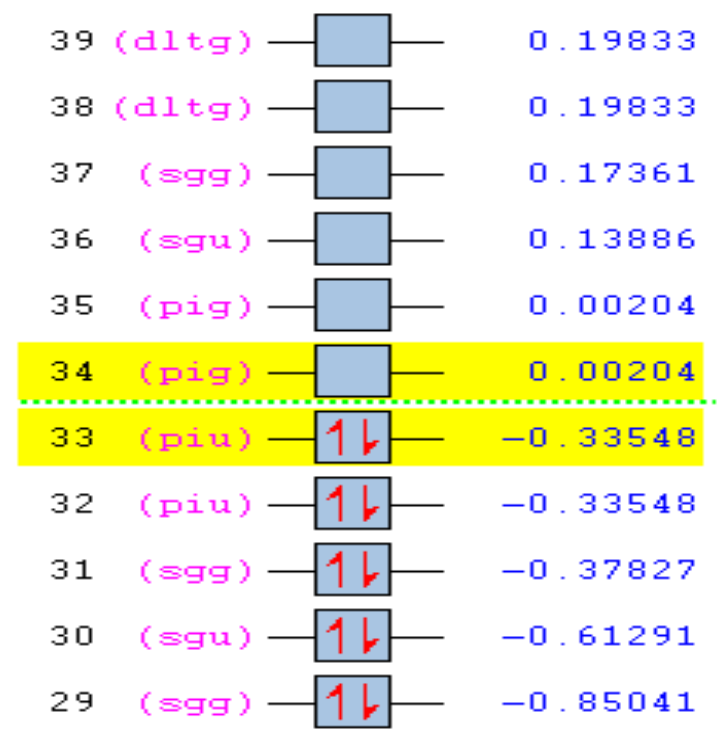

Figure 3.4: HOMO and LUMO energy levels in the arsenic molecule with MP2 levels of approximation using the basis set $6-311 \mathrm{G}(3 \mathrm{df})$

From Figure 3.4, we found the energy of highest occupied molecular orbital and that of lowest unoccupied molecular orbital in the arsenic molecule $\left(\mathrm{As}_{2}\right)$ to be $\mathrm{E}_{\mathrm{H}}=-9.13 \mathrm{eV}$ and $\mathrm{E}_{\mathrm{L}}=0.06 \mathrm{eV}$ respectively, which gives the value of HOMOLUMO energy gap of arsenic molecule $\left(\mathrm{As}_{2}\right)$ to be $9.19 \mathrm{eV}$. Our result also shows that the value of the HOMO-LUMO energy gap of arsenic molecule $\left(\mathrm{As}_{2}\right)$ is almost independent of choice of basis sets as well as levels of approximation.

\section{(v) EFG parameters for arsenic molecule ( $\left.\mathrm{As}_{2}\right)$}

For the calculations of nuclear quadrupole interaction (NQI) parameters of the arsenic molecule $\left(\mathrm{As}_{2}\right)$, we have chosen EFG parameters considering optimized geometry (i.e. the distance between two arsenic atoms to be $2.15 \AA$ ). Our calculated values of $\left|V_{x x}\right|$ and $\left|V_{y y}\right|$ are equal giving the asymmetry parameter, defined as;

$$
\eta=\frac{\left|V_{y y}\right|-\left|V_{x x}\right|}{\left|V_{z z}\right|}
$$

to be zero for arsenic molecule $\left(\mathrm{As}_{2}\right)$. The equality of $\left|V_{x x}\right|$ and $\left|V_{y y}\right|$ shows the spherical symmetry of charge distribution of the arsenic molecule $\left(\mathrm{As}_{2}\right)$. We have also calculated the nuclear quadrupole coupling constant $\left(e^{2} Q q / h\right)$ for arsenic molecule $\left(\mathrm{As}_{2}\right)$ taking the value of $Q$ to be 0.3 barn [5]. In the expression $e^{2} Q q / h, e$ is the electronic charge, $Q$ is nuclear quadrupole moment, $h$ is plancks constant, and $\left|V_{z z}\right|=q e$. With HF, MP2, and QCISD values of $\left|V_{z z}\right|$ obtained with 6-311G(3df) basis set, we have estimated the HF, MP2, and QCISD values of $\left(e^{2} Q q / h\right)$ for arsenic molecule $\left(\mathrm{As}_{2}\right)$ to be 237.267, 239.008, and 239.008 MHz respectively. The closeness of the HF, MP2, and QCISD values of nuclear quadrupole coupling constant $\left(e^{2} Q q / h\right)$ for arsenic molecule
$\left(\mathrm{As}_{2}\right)$ clearly indicates that the electron correlation effect do not contribute significantly for the determination of EFG parameters for arsenic molecule $\left(\mathrm{As}_{2}\right)$. With this remark, we conclude the nuclear quadrupole coupling constant $\left(e^{2} Q q / h\right)$ for arsenic molecule $\left(\mathrm{As}_{2}\right)$ to be 237.267, 239.008, and $239.008 \mathrm{MHz}$ respectively in HF, MP2, and QCISD levels of approximation, which is close to the previously reported value of $247 \mathrm{MHz}$ [16] within 4\%. By using this value of nuclear quadrupole coupling constant, we have calculated the nuclear quadrupole frequency $v_{Q}[=]$; to be 118.634, 119.504, and $119.504 \mathrm{MHz}$ with $\mathrm{I}=3 / 2$ in HF, MP2, and QCISD levels of approximation respectively.

\section{CONCLUSION AND OUTLOOKS}

In the present work, we have performed the ground state energy calculations for arsenic atom, its ions and molecule. We have also performed the first-principles calculation to estimate the electron affinity and ionization energy of arsenic atom. With the MP2 levels of approximation using the basis set 6-31G, we have estimated the electron affinity for arsenic atom to be $0.81 \mathrm{eV}$, which is within $1.3 \%$ as compared to the experimental value of $-0.80 \mathrm{eV}$. We have also estimated the values of the ionization energies for the arsenic atom by considering different levels of ionization i.e. $\mathrm{As}^{+}$to $\mathrm{As}^{+10}$. Our estimated HF, MP2 and DFT values of the first to tenth ionization energies for the arsenic atom agree to the corresponding experimental values within $6 \%$. This shows that our estimated values are as expected and within the acceptable limit as compared to the experimental values. The first principles calculation has also been carried out to study the equilibrium configuration of arsenic molecule $\left(\mathrm{As}_{2}\right)$. We have estimated the bond length and binding energy arsenic molecule $\left(\mathrm{As}_{2}\right)$ to be $2.15 \AA$ and3.65 eV in MP2 levels of calculation with the basis set $6-311 \mathrm{G}(3 \mathrm{df})$, which agree to the experimental values of 2.44 $\AA$ and $3.61 \mathrm{eV}$ within $13 \%$ and $1 \%$ respectively. Our study has been further extended to calculate electrostatic potential for arsenic molecule $\left(\mathrm{As}_{2}\right)$, whose values at global maxima and minima are found to be $0.30 \mathrm{eV}$ and $-0.20 \mathrm{eV}$ respectively. We have also performed the Basis Set Superimposition Error calculations using Counterpoise method for various basis sets and levels of approximation in ordered to obtain the corrected binding energy. Our result shows that counterpoise method gives more close value of binding energy to the experimental value than the corresponding value without counterpoise method, indicating that while performing atomic calculations it is necessary to account for the BSSE. Also our results show that the BSSE energy is higher in the smaller basis sets and that is lower in the larger basis sets. This indicates that BSSE calculation is necessary for lower basis sets. We have also calculated HOMO-LUMO energy gap for the arsenic molecule $\left(\mathrm{As}_{2}\right)$ in HF, MP2 and QCISD levels of approximation using different basis sets with NBO analysis method. The value of HOMO-LUMO energy gap of arsenic molecule $\left(\mathrm{As}_{2}\right)$ is 
found to be around $9.00 \mathrm{eV}$ in all levels of approximation with different basis sets indicating that the value of the HOMOLUMO energy gap is almost independent of choice of basis sets as well as levels of approximation. The HF, MP2 and QCISD calculations carried out to estimate the electric field gradient (EFG) parameters, i.e., the largest principal component $(q)$ of EFG tensor and asymmetry parameter $(\eta)$ for the excited nuclear state in arsenic molecule $\left(\mathrm{As}_{2}\right)$ shows that these values for the EFG parameters are close to each other, indicating that electron correlation effects do not contribute significantly to the determination of EFG parameters and asymmetry parameter to be essentially zero.

\section{ACKNOWLEDGEMENTS}

We thank Mr. D. B. Karki of the International Centre for Theoretical Physics (ICTP) Italy, for reviewing the manuscript and making a number of important recommendations for its improvement.

\section{REFERENCES}

[1] K. S. Bhatia and W. E. Jones, Can. J. Phys. 49, 1773 (1971).

[2] J. CHE, K. JHANG and X. XIE, Journal of Electron Spectroscopy and Related Phenomenon 80, 169172 (1996).

[3] K. P. Huber and G. Herzberg, Constants of diatomic molecules, Van Nostrad Rcinhold, New York, (1979).

[4] K. Balasubramanian, Che. Phys. Lett. 171 (1990).

[5] J. Emsley, The Elements, University Press, Oxford (1989).

[6] L. Umaralikhan et al, J. Comp. Methods in Molecular Design, 4 25-32 (2014).

[7] Cossairt, B. C. Cossairt and C. C. Cummins, Journal of the American Chemical Society, 42, 131 (2009).

[8] J. M. Thijssen, Computational Physics, Cambridge University Press, Cambridge, first edition (1999).

[9] D. A. McQuarrie, Quantum Chemistry, University Science Books, Sausalito (1983).

[10] K. Ohno et al, computational Material Science, Springer Verlag, Berlin (1999).

[11] C. Kittel, Introduction to Solid State Physics, John Wiley and Sons, Inc., Singapore, fourth edition (1971).

[12] M. J. Frisch et al Gaussian 03, Revision C.01, Gaussian, Inc., Wallingford CT (2004).

[13] K. Fukui, Science, 218 (1982).

[14] D. B. Karki and N. P. Adhikari, Int. J. Mod. Phys. 28 (2014).

[15] D. B. Karki and N. P. Adhikari, arXiv. Conds. Mtrl. Sci 6644 (2014).

[16] F. Leung, S. A. Cooke, and M. C. L. Gerry, J. Mol. Spect. 6, 123 (2006). 